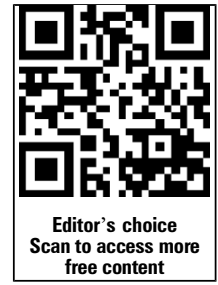

- Additional material is published online only. To view please visit the journal online (http://dx.doi.org/10.1136/ annrheumdis-2014-207169).

${ }^{1}$ Rheumatology Unit, The Queen Elizabeth Hospital, Woodville, South Australia ${ }^{2}$ University of Adelaide, The Health Observatory, Adelaide, South Australia

${ }^{3}$ Royal North Shore Hospital, Institute of Bone and Joint Research, St Leonards, New South Wales, Australia ${ }^{4}$ Menzies Research Institute, Hobart, Tasmania, Australia ${ }^{5}$ Rheumatology Unit, Royal Adelaide Hospital, Adelaide, South Australia

\section{Correspondence to} Dr Catherine Hill,

Rheumatology Unit, The Queen Elizabeth Hospital, 28 Woodville Road Woodville, SA 5011, Australia;

catherine.hill@health.sa.gov.au

Received 17 December 2014 Revised 17 August 2015 Accepted 19 August 2015 Published Online First 9 September 2015

\section{(1) CrossMark}

\section{SLinked}

- http://dx.doi.org/10.1136/ annrheumdis-2015-208329

To cite: Hill $C L$, March $L M$, Aitken $\mathrm{D}$, et al. Ann Rheum Dis 2016;75:23-29.

\title{
Fish oil in knee osteoarthritis: a randomised clinical trial of low dose versus high dose
}

\author{
Catherine L Hill, ${ }^{1,2}$ Lynette M March, ${ }^{3}$ Dawn Aitken, ${ }^{4}$ Susan E Lester, ${ }^{1}$ \\ Ruth Battersby, ${ }^{1}$ Kristen Hynes, ${ }^{4}$ Tanya Fedorova, ${ }^{3}$ Susanna M Proudman, ${ }^{5}$ \\ Michael James, ${ }^{5}$ Leslie G Cleland, ${ }^{5}$ Graeme Jones ${ }^{4}$
}

\section{ABSTRACT}

Objectives To determine whether high-dose fish oil is superior to low-dose supplementation for symptomatic and structural outcomes in knee osteoarthritis (OA). Methods A randomised, double-blind, multicentre trial enrolled 202 patients with knee OA and regular knee pain. They were randomised 1:1 to high-dose fish oil (4.5 g omega-3 fatty acids) $15 \mathrm{~mL} /$ day or (2) low-dose fish oil (blend of fish oil and sunola oil; ratio of 1:9, $0.45 \mathrm{~g}$ omega-3 fatty acids) $15 \mathrm{~mL} /$ day. The primary endpoints were Western Ontario and McMaster Universities Arthritis Index (WOMAC) pain score at 3, 6, 12 and 24 months, and change in cartilage volume at 24 months. Secondary outcomes included WOMAC function, quality of life, analgesic and non-steroidal antiinflammatory drug use and bone marrow lesion score. Results Although there was improvement in both groups, the low-dose fish oil group had greater improvement in WOMAC pain and function scores at 2 years compared with the high-dose group, whereas between-group differences at 1 year did not reach statistical significance. There was no difference between the two groups in cartilage volume loss at 2 years. For other secondary endpoints, there was no difference between the two groups at 2 years.

Conclusions In people with symptomatic knee OA, there was no additional benefit of a high-dose fish oil compared with low-dose fish oil. The combination comparator oil appeared to have better efficacy in reducing pain at 2 years, suggesting that this requires further investigation.

Trial registration number Australian New Zealand Clinical Trials Registry (ACTRN 12607000415404).

\section{INTRODUCTION}

Osteoarthritis (OA) is a major cause of disability in older persons. ${ }^{1}$ Current medical treatment is confined to symptom control with paracetamol and/or non-steroidal anti-inflammatory drugs (NSAIDs), as well as physical therapy and weight loss. ${ }^{2}$ Although NSAIDs transiently reduce knee OA pain, due to the adverse effects, American College of Rheumatology (ACR) guidelines recommend intermittent use only. ${ }^{2}$ A systematic review of fish oil use in rheumatoid arthritis (RA) reported decreased NSAID use with fish oil, ${ }^{3}$ and a randomised controlled trial (RCT) of high-dose fish oil in recent-onset RA demonstrated a higher remission rate. ${ }^{4}$ Synovial inflammation is associated with severity of pain in knee $\mathrm{OA}^{5-7}$ and has been variably associated with cartilage loss. ${ }^{7} 8$
Since synovitis and cartilage degradation are common to both RA and OA, it is possible that fish oil may be useful in OA.

Eicosapentaenoic acid (EPA) and docosahexaenoic acid (DHA), the main omega-3 fatty acids in fish oil, decrease synthesis of the cyclooxygenase omega-6 fatty acid metabolite, prostaglandin E2 also a target of NSAID action. EPA and DHA are also precursors of the E-resolvins and D-resolvins that suppress inflammatory cytokine production and act to resolve inflammation. ${ }^{9}$ In vitro experiments and animal OA models suggest potential benefit of EPA/DHA in OA, although few studies have been undertaken. ${ }^{10-13}$ In healthy adults, higher baseline dietary intake of monounsaturated fats and n-6 fatty acids has been associated with increased bone marrow lesions (BMLs) on MRI 10 years later, but no significant effect on cartilage volume. ${ }^{14}$ Data from the MOST study $(n=472)$ showed a negative association between total n-3 fatty acid levels and patellofemoral cartilage loss, but no association with synovitis or tibiofemoral cartilage loss. ${ }^{15}$ Neither study included n-3 fatty acids supplementation.

Community use of omega-3 supplements is widespread. An Australian study of 260000 people reported $32.6 \%$ had taken omega-3 supplements within the past four weeks with presence of OA being positively correlated with use. ${ }^{16}$ However, most people are taking a low median daily dose of $1 \mathrm{~mL}$ of fish oil, which contains approximately $30 \%(0.3 \mathrm{~g})$ EPA+DHA. ${ }^{17}$ Studies in RA and other inflammatory diseases have indicated that the antiinflammatory dose of fish oil requires delivery of $\geq 2.7 \mathrm{~g}$ of $\mathrm{EPA}+\mathrm{DHA}$ daily, ${ }^{10}$ requiring approximately $10 \mathrm{~mL}$ of standard fish oil per day. Therefore, most people who self-medicate with fish oil are generally taking much less than the antiinflammatory dose.

The aim of this study was to compare the effects of an anti-inflammatory dose of fish oil with a lower dose of fish oil (not considered to be in the anti-inflammatory range), in a double-blind RCT of knee OA. The comparator of low-dose fish oil was chosen for masking of high-dose fish oil and to allow compliance with recommendations on EPA + DHA intake for cardiovascular prevention. ${ }^{18}$ The study hypothesis was that high-dose fish oil would have superior efficacy to low-dose fish oil for symptomatic and structural outcomes in people with knee OA. 


\section{METHODS}

\section{Study design}

We undertook a double-blind, randomised trial. Participants were recruited from the community through general media advertising and rheumatology databases at three Australian centres (August 2007-September 2009). Further details of the study protocol are available in online supplementary file.

\section{Participants}

Participants were $>40$ years with clinical knee OA defined using ACR criteria $^{19}$ and visual analogue scale knee pain score $>20 \mathrm{~mm}$ (0-100 mm scale). Exclusion criteria included severe radiographic knee OA in index knee (grade 3 radiographic joint space narrowing using Osteoarthritis Research Society International atlas ${ }^{20}$ ), dementia or inability to give informed consent, pregnancy or lactation, planned knee replacement surgery, long-term use ( $\geq 6$ months) of high-dose fish oil (equivalent to $15 \mathrm{~mL}$ of oil) and contraindications to MRI.

\section{Run-in, randomisation and masking}

Prior to randomisation, a 4-week run-in period with similarly flavoured oil (citrus-flavoured sunola oil), $15 \mathrm{~mL} /$ day, was performed to exclude participants intolerant of liquid oil. Participants who tolerated oil during the run-in period were randomly allocated to one of two treatment arms: high-dose or low-dose fish oil, $15 \mathrm{~mL}$ per day. High-dose fish oil contained EPA $18 \%$ and DHA 12\%, supplying $4.5 \mathrm{~g}$ EPA+DHA per day. The comparator oil was a blend of low-dose fish oil and higholeic sunola oil in a ratio of $1: 9$, supplying $0.45 \mathrm{~g}$ EPA+DHA per day, equivalent to 1.5 standard $1 \mathrm{~g}$ fish oil capsule daily. Both oils were flavoured with citrus oils and provided in identical dark $500 \mathrm{~mL}$ bottles. The oils, blending, masking and bottling were provided by Melrose Health, Victoria, Australia. Study oil bottles were returned at each study visit and volume of unconsumed oil was measured to assess compliance. Participants were provided with paracetamol $(500 \mathrm{mg})$ tablets with instructions that they could safely use up to $8 /$ day.

The computer-generated random allocation sequence and subsequent allocation was performed centrally at one pharmacy with stratification for study site. Participants and staff involved in patient care and assessment of MRI remained blinded throughout the study.

\section{Outcome measures}

Primary outcomes were knee-specific pain scales (Western Ontario and McMaster Universities Arthritis (WOMAC) index) at 3, 6, 12 and 24 months and change in cartilage volume on MRI at 24 months. Secondary outcome measures were WOMAC function, quality of life, analgesic and NSAID use, change in BML score and safety outcomes.

The WOMAC numerical rating scale (NRS) 3.1 index for knee pain and function, measured on a 10-point numerical scale, ${ }^{21}$ and the Assessment of Quality of Life utility instrument, which has been validated in both the general population and patients with $\mathrm{OA},{ }^{22}$ were measured 3 monthly. Analgesic use was measured using pill counts for paracetamol and daily diary for NSAIDs, using NSAID equivalence scores. ${ }^{23}$

MRIs of the study knee were performed at baseline and 2 years with $1.5 \mathrm{~T}$ whole-body $\mathrm{MR}$ unit using a commercial receive-only extremity coil. The MRI sequence was a T1-weighted, fat-suppressed, three-dimensional (3D) gradient recall acquisition in the steady state; flip angle $55^{\circ}$; repetition time $58 \mathrm{~ms}$; echo time $12 \mathrm{~ms}$; field of view $16 \mathrm{~cm}$; 60 partitions;
$512 \times 192$ matrix; one acquisition time $11 \mathrm{~min}, 56 \mathrm{~s}$. Sagittal images were obtained at a partition thickness of $1.5 \mathrm{~mm}$ and an in-plane resolution of $0.31 \times 0.83 \mathrm{~mm}(512 \times 192$ pixels $)$.

Individual cartilage plate volumes (medial tibia, lateral tibia and patella) were isolated from the total volume by manually drawing disarticulation contours around the cartilage boundaries on a section-by-section basis. Data were then resampled by means of bilinear and cubic interpolation (area of 312 and $1.5 \mathrm{~mm}$ thickness, continuous sections) for the final $3 \mathrm{D}$ rendering. There was one trained reader, blinded to treatment allocation and clinical data, with coefficient of variation of $2.1-2.6 \% .^{24}$

BMLs were assessed on a proton density-weighted fat saturation 2D fast spin echo sequence in the sagittal plane. They were defined as areas of increased signal adjacent to the subcortical bone at the medial tibial, medial femoral, lateral tibial, lateral femoral, superior patella and inferior patella sites. BMLs were scored by measuring the maximum area of the lesion $\left(\mathrm{mm}^{2}\right)$ at baseline and follow-up. ${ }^{25}$ There was one trained reader, blinded to treatment allocation and clinical data, with intraclass correlation coefficient of $0.97 .^{25} \mathrm{BML}$ size at all six sites was summed to create total BML size at each time point. A meaningful BML change was considered to be $140 \mathrm{~mm}^{2}$ change in either direction, which corresponds to a one-unit change in WOMAC pain score. ${ }^{25} 26$

MRI scans at both baseline and end of study were available for cartilage reading $(n=116)$, and BML reading $(n=110)$ as only participants with readable scans at baseline and 24 months were included. All participants from one site were excluded from MRI analysis due to inconsistent MRI sequencing from baseline to 24 months $(n=51)$. Further participants were excluded due to loss to follow-up, non-readable MRI, screws in the knee, incorrect sequence at one time point or incorrect knee scanned at one time point.

\section{Serum fatty acid analysis}

Fasting serum phospholipid fatty acid from two sites (Adelaide and Sydney; $n=150$ ) were measured at each clinic visit by capillary gas chromatography. ${ }^{27}$

\section{Sample size}

Sample sizes of 100 per treatment group were selected based on power calculations for longitudinal data with six treatment visits, $\alpha=0.05, \beta=0.2$, an attrition rate of $5 \%$ per visit and a standardised treatment effect at the end of the study of 0.4 (ie, a medium effect).

\section{Statistical analysis}

Primary hypotheses were tested using intention-to-treat (ITT) analysis, with secondary per protocol (PP) analysis of those who finished the 24-month visit taking study oil.

Analysis of outcomes at each visit was performed by constrained longitudinal data analysis, ${ }^{28}$ using $\mathrm{R}$ statistical software. ${ }^{29}$ Mixed effects models were estimated, with both patient and centre as random effects, and an autocorrelation error structure using the nlme library. ${ }^{30}$ WOMAC scores were analysed from 20 multiply imputed data sets, imputed using the Amelia library. ${ }^{31}$ Treatment effects for normally distributed variables were expressed as mean differences. Non-normally distributed variables were log-transformed as appropriate, and treatment effects for these variables were expressed as ratios. Relative risks were estimated for dichotomous outcomes. Causal mediation analysis, for the influence of weight gain on WOMAC outcomes, was performed using the $\mathrm{R}$ library mediation. ${ }^{32}$ 


\section{RESULTS}

\section{Participants}

A total of 351 participants were screened (figure 1). A further 49 participants failed to complete the run-in period. Two-hundred and two participants were randomised to either arm. There was one protocol violation following randomisation. Although 54 (26.7\%) discontinued the intervention, follow-up was $84 \%$ at 24 months as consenting participants were evaluated at 12 and 24 months irrespective of continuing the intervention. At baseline, participants in each group were well-matched, except for gender (table 1). There were more female participants randomised to the high-dose fish oil group $(59 \%)$ compared with low-dose fish oil group $(40 \%, \mathrm{p}<0.01)$. The majority had evidence of radiographic OA (194/202; 96\%).

Withdrawal from therapy was higher in the high-dose compared with the low-dose group (35\% vs $20 \%)$ and occurred earlier (median time to cessation 3 vs 7.5 months). Reasons for withdrawals are shown in figure 1 .

\section{Pain and function}

The low-dose fish oil group had lower pain scores at 18 and 24 months and better functional limitation scores at 24 months compared with the high-dose group (figure 2D, E and table 2). These differences were demonstrated in both ITT and PP analyses (table 2). Adjustment for gender had little or no effect on the outcomes (table 2).

There was no difference between the two groups in the use of paracetamol or NSAIDs (see online supplementary table S1, figure S1 and supplementary data) during the study, nor any difference in quality of at any time point (figure $2 \mathrm{~F}$ ).

\section{MRI results}

There was no statistically significant change in total cartilage volume from baseline to 24 months and no difference between the groups in the changes over 24 months (table 3 ). There was no difference in the proportion of participants who had a clinically significant change in BML over 24 months (table 3).

\section{Other outcomes}

There was no change in serum $\mathrm{C}$ reactive protein levels over time and no difference between groups (data not shown).

Both treatment groups, on average, gained a small amount of weight (see online supplementary table S2 and supplementary data), with significantly greater weight gain in the high-dose group $(p<0.05)$. As weight gain is a potential mediator of effects on pain and function in knee $\mathrm{OA},{ }^{34-36}$ it is plausible that the increased weight gain observed in the high-dose group may have contributed to their poorer WOMAC scores. A post hoc causal

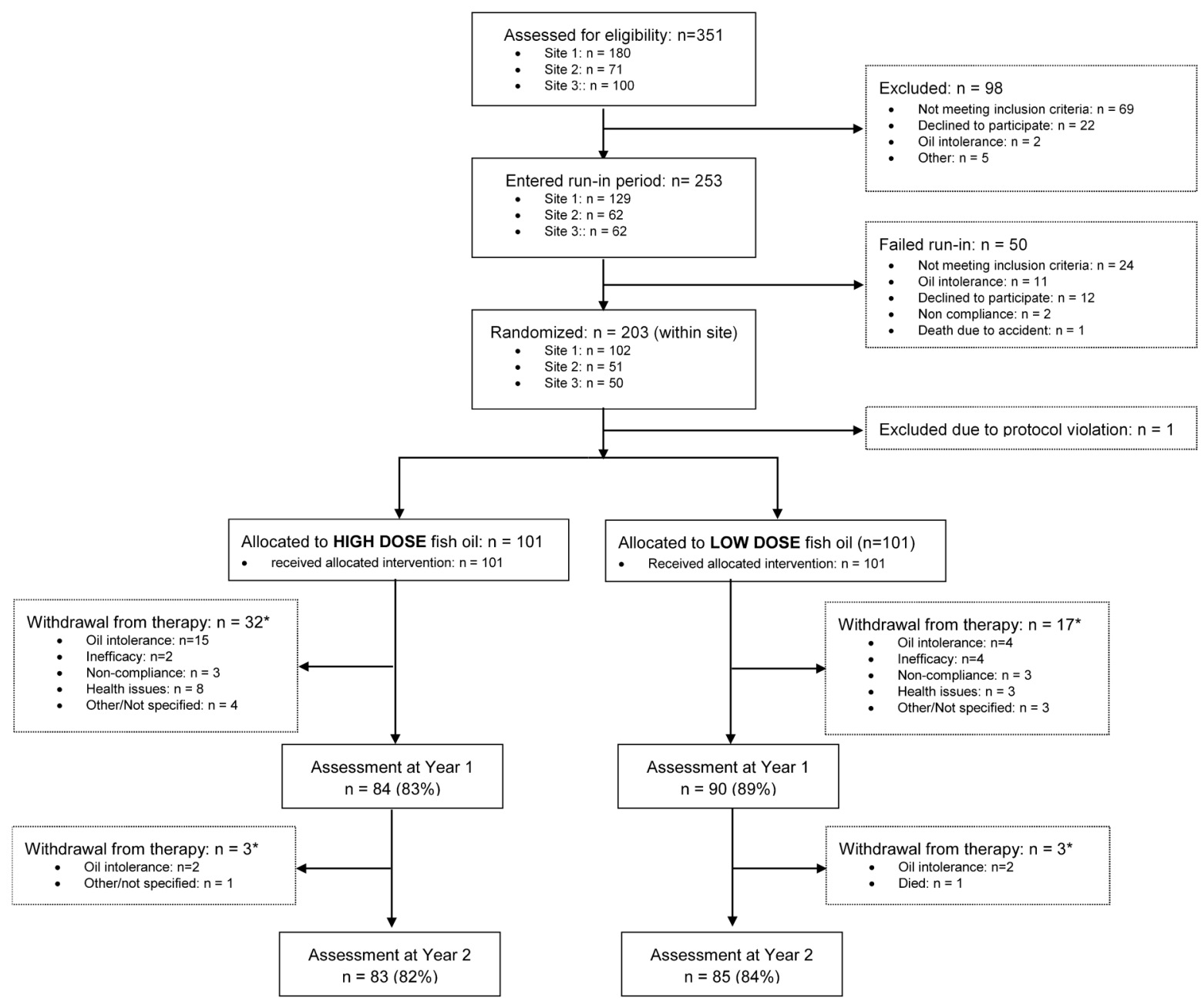

*Not all withdrawn from study assessment

Figure 1 Flow diagram of participant recruitment and completion. 


\begin{tabular}{|c|c|c|c|}
\hline Characteristic & Low-dose fish oil & High-dose fish oil & $\mathrm{p}$ Value \\
\hline $\mathrm{N}$ & 101 & 101 & \\
\hline Gender (\% female) & 40 & 59 & $<0.01$ \\
\hline BMI $\left(\mathrm{kg} / \mathrm{m}^{2}\right)$ : mean (SD) & $29(4)$ & $29(5)$ & 0.67 \\
\hline WOMAC* pain: mean (SD) & $15(9)$ & $16(9)$ & 0.35 \\
\hline Quality of life (AQoL-4D): mean (SD) & $0.77(0.27)$ & $0.74(0.25)$ & 0.50 \\
\hline NSAID use (\%) & 34 & 30 & 0.54 \\
\hline Radiographic knee OA (OARSI\%) ${ }^{20}$ & 96/101 (95\%) & $98 / 101(97 \%)$ & 0.47 \\
\hline Total OARSI joint space narrowing :mean (SD) & $1.7(0.1)$ & $1.8(0.1)$ & 0.62 \\
\hline Total OARSI osteophyte score: mean (SD) & $2.0(0.3)$ & $2.3(0.2)$ & 0.42 \\
\hline CRP: median (IQR) & $1.5(2.2)$ & $1.7(2.3)$ & 0.43 \\
\hline \multicolumn{4}{|l|}{ Plasma omega-3 fatty acids } \\
\hline Plasma EPA (20:5n-3): mean (SD) & $1.3(0.7)(n=95)$ & $1.5(1.1)(n=94)$ & 0.17 \\
\hline Plasma DHA (22:6n-3): mean (SD) & $3.4(1.0)(n=95)$ & $3.5(1.2)(n=94)$ & 0.75 \\
\hline
\end{tabular}

mediation analysis determined that weight, as a mediator variable, contributed $<1 \%$ to the average difference between the high-dose and low-dose treatments (over the six treatment visits) for either pain $(p=0.88)$ or function $(p=0.89)$. It is therefore unlikely that the between-group differences in WOMAC outcomes can be attributed to differences in weight gain.

\section{Compliance}

Assessed by measuring the oil volume in returned bottles, compliance was $>80 \%$ in both groups. Both groups had increases from baseline in plasma EPA and DHA with the high-dose group having substantially larger increases, consistent with compliance with study oil (figure 2A-C).

\section{Success of blinding}

At the end of the study, $52 \%$ of participants were unsure which group to which they had been allocated (50\% high dose, 50\% low dose). Of the remaining who thought they knew which group they were allocated, only 57\% answered correctly, suggesting that blinding had been well maintained.

\section{Adverse events}

Adverse events were common and did not occur more frequently in either group (table 4). Serious adverse events were primarily non-elective hospital admissions. There was a sudden cardiac death in the low-dose group, considered unrelated to the intervention. There were no significant bleeding or thrombosis complications in either group. Although gastrointestinal adverse events were equally common in each group, this led to greater discontinuation of treatment in the high-dose compared with the low-dose group $(16.8 \%$ vs $5.9 \%$; $\mathrm{p}<0.015)$.

\section{DISCUSSION}

This double-blinded randomised clinical trial demonstrated that ingestion of low-dose fish oil (in combination with sunola oil) resulted in better pain and function scores at 18 and 24 months compared with high-dose fish oil. This difference occurred with no change in the use of analgesics or NSAIDs over 24 months. There was no difference in structural outcomes of cartilage volumes and BMLs over 24 months. The study showed no benefit of high-dose fish oil over low-dose fish oil, which was the primary hypothesis of the study. Unexpectedly, the lower dose fish oil group had less pain and better function than the high-dose group. The reasons for this unanticipated result remain unclear. There was greater weight gain in the high-dose group compared with the low-dose group, which may contribute to higher pain scores. However, a post hoc mediator analysis demonstrated this differential weight gain did not influence the difference in pain and function seen between the two groups. The small difference in weight gain is itself difficult to explain, as both groups consumed equivalent volumes of oil, with similar caloric intake.

There was no group difference at 24 months in change in cartilage volume or BMLs. Although MRI data from one site could not be used due to inconsistent MRI sequences, there was no imbalance in the groups due to stratification of randomisation by study site, and there was good follow-up (84\%) for MRI data from the other two sites. However, there was loss of power for these endpoints, so it is not possible to make firm conclusions regarding structure modification from our findings.

The comparator oil, which contained predominantly sunola oil, was not expected to have any therapeutic effect. It is low in saturated fatty acids, n- 6 fatty acids and n-3 fatty acids, and is predominantly non-essential, monounsaturated oleic acid (n-9). A previous 6-month RCT in OA comparing cod liver oil (rich in omega-3 fatty acids) and olive oil (rich in n-9 fatty acids) demonstrated no difference between the groups after 6 months. ${ }^{37}$ However, in contrast to olive oil, sunola oil is not rich in polyphenols with which anti-inflammatory actions have been associated. ${ }^{38} 39$ One possible explanation could be that 
Figure 2 Mean n-3 fatty acids and osteoarthritis outcomes over the study duration (intention-to-treat patients) for high-dose compared with low-dose fish oil treatment. Vertical error bars represent the SE of the mean. n-3 fatty acids ((A) eicosapentaenoic acid (EPA);

(B) docosahexaenoic acid (DHA); and

(C) total $n-3)$ ) were expressed as a percentage of total fatty acids, and were significantly increased in high-dose compared with low-dose patients at all treatment visits. Western Ontario and McMaster Universities Arthritis Index (WOMAC) outcomes were (D). Pain (numerical rating scale (NRS) 3.1 0-50 scale) and (E) function (NRS 3.1 0-170 scale). Both were significantly higher in the high-dose patients at the end of study $(<0.10$, $\left.{ }^{*} p<0.05,{ }^{*} p<0.01\right)$. There were no significant differences between the two treatment groups for quality of life scores ((F) AQoL-4D).
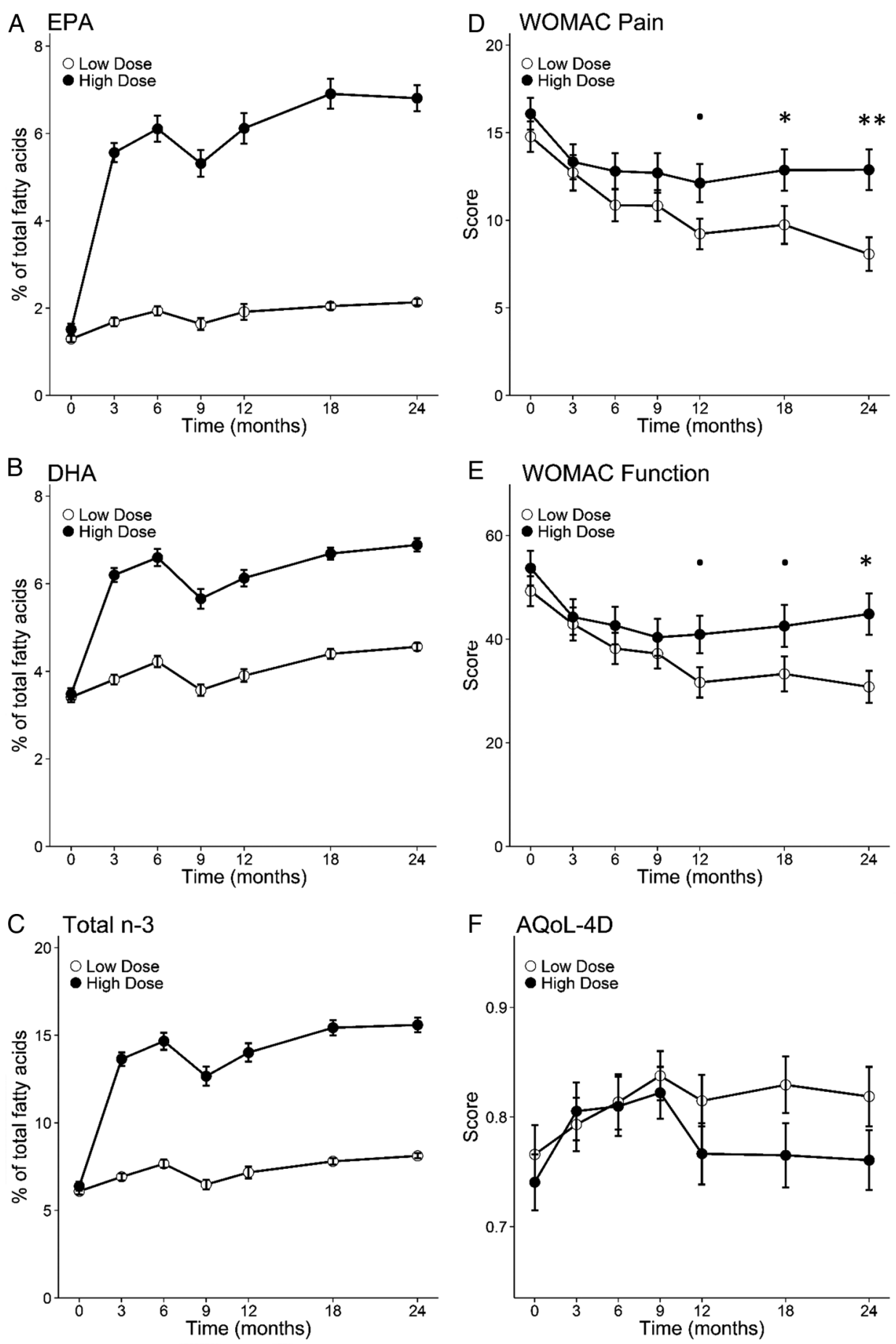

Table 2 Difference in Western Ontario and McMaster Universities Arthritis Index (WOMAC) outcomes between high-dose and low-dose fish oil at 1 and 2 years, respectively

\begin{tabular}{|c|c|c|c|c|c|c|c|c|}
\hline \multirow{3}{*}{ Outcome } & \multicolumn{4}{|c|}{ Intention-to-treat } & \multicolumn{4}{|l|}{ Per protocol } \\
\hline & \multicolumn{4}{|c|}{ High dose-low dose } & \multicolumn{4}{|c|}{ High dose-low dose } \\
\hline & \multicolumn{2}{|c|}{1 year $(n=101)$} & \multicolumn{2}{|c|}{2 years $(n=101)$} & \multicolumn{2}{|c|}{1 year $(n=80)$} & \multicolumn{2}{|c|}{2 years $(n=65)$} \\
\hline WOMAC Pain & $2.3(1.2)$ & 0.06 & $3.3(1.3)$ & 0.009 & $3.3(1.2)$ & 0.007 & $4.1(1.2)$ & 0.001 \\
\hline Gender adjusted & $2.1(1.2)$ & 0.081 & $3.1(1.3)$ & 0.014 & $3.1(1.2)$ & 0.009 & $4.0(1.2)$ & 0.001 \\
\hline WOMAC function & $6.5(3.7)$ & 0.08 & $8.5(4.0)$ & 0.032 & $8.4(3.6)$ & 0.019 & $11.6(3.7)$ & 0.002 \\
\hline
\end{tabular}


Table 3 Changes in MRI cartilage volume and bone marrow lesion area over 2 years of fish oil treatment

\begin{tabular}{|c|c|c|c|c|c|c|}
\hline & \multicolumn{3}{|c|}{ Intention to treat } & \multicolumn{3}{|c|}{ Per protocol } \\
\hline & Low dose & High dose & $\mathrm{p}$ Value & Low dose & High dose & $\mathrm{p}$ Value \\
\hline $\mathrm{N}$ & 56 & 59 & & 53 & 45 & \\
\hline Decrease & $3(5 \%)$ & $8(14 \%)$ & $0.21^{*}$ & $2(4 \%)$ & $5(11 \%)$ & $0.09^{*}$ \\
\hline No change & $47(84 \%)$ & $42(71 \%)$ & & $46(87 \%)$ & $31(69 \%)$ & \\
\hline Increase & $6(11 \%)$ & $9(15 \%)$ & & $5(10 \%)$ & $9(20 \%)$ & \\
\hline Decrease & $5(9 \%)$ & $7(13 \%)$ & $0.23^{*}$ & $5(10 \%)$ & $6(14 \%)$ & $0.41^{*}$ \\
\hline No change & $48(87 \%)$ & $42(76 \%)$ & & $45(87 \%)$ & $33(77 \%)$ & \\
\hline Increase & $2(4 \%)$ & $6(11 \%)$ & & $2(4 \%)$ & $4(9 \%)$ & \\
\hline
\end{tabular}

${ }^{*} p$ Values refer to comparisons between fish oil treatment groups.

tLeast significant change ${ }^{33}$ was considered a change of $>8 \%$ in either direction.

$\ddagger$ Clinically significant was considered a change $>140 \mathrm{~mm}^{2}$ in either direction, which corresponds to a one-unit change in Western Ontario and McMaster Universities Arthritis Index score. $^{25}$

\begin{tabular}{|c|c|c|}
\hline & Low-dose fish oil & High-dose fish oil \\
\hline Hospitalisations & $37(36.6 \%)$ & $38(37.6 \%)$ \\
\hline Infection (all) & 66 (65.3\%) & 71 (70.3\%) \\
\hline Respiratory & $46(45.5 \%)$ & $51(50.5 \%)$ \\
\hline Ear & $1(1.0 \%)$ & $4(4.0 \%)$ \\
\hline Other & $19(18.8 \%)$ & $16(15.8 \%)$ \\
\hline Gastrointestinal (all) & $62(61.4 \%)$ & $67(66.3 \%)$ \\
\hline Upset & $26(25.7 \%)$ & $15(14.9 \%)$ \\
\hline Reflux & $12(11.9 \%)$ & $17(16.8 \%)$ \\
\hline Nausea & $13(12.9 \%)$ & $19(18.8 \%)$ \\
\hline Diarrhoea & $5(5.0 \%)$ & $8(7.9 \%)$ \\
\hline Intolerance/other & $6(5.9 \%)$ & $8(7.9 \%)$ \\
\hline Bleeding (all) & $4(4.0 \%)$ & $1(1.0 \%)$ \\
\hline Epistaxis & $1(1.0 \%)$ & $0(0.0 \%)$ \\
\hline Haemarthrosis & $0(0.0 \%)$ & $1(1.0 \%)$ \\
\hline Postoperative & $1(1.0 \%)$ & $0(0.0 \%)$ \\
\hline Rectal & $1(1.0 \%)$ & $0(0.0 \%)$ \\
\hline Minor & $1(1.0 \%)$ & $0(0.0 \%)$ \\
\hline Thrombosis (all) & $1(1.0 \%)$ & $1(1.0 \%)$ \\
\hline Superficial leg vein & $1(1.0 \%)$ & $0(0.0 \%)$ \\
\hline Deep vein thrombosis & $0(0.0 \%)$ & $1(1.0 \%)$ \\
\hline Cancer (all) & $9(8.9 \%)$ & $12(11.9 \%)$ \\
\hline Breast cancer & $1(1.0 \%)$ & $1(1.0 \%)$ \\
\hline Prostate cancer & $1(1.0 \%)$ & $0(0.0 \%)$ \\
\hline Non-melanotic skin Ca & $6(5.9 \%)$ & $9(8.9 \%)$ \\
\hline Melanoma & $1(1.0 \%)$ & $0(0.0 \%)$ \\
\hline Other cancer & $0(0.0 \%)$ & $2(2.0 \%)$ \\
\hline Cardiovascular (all) & $16(15.8 \%)$ & $18(17.8 \%)$ \\
\hline Sudden cardiac death & $1(1.0 \%)$ & $0(0.0 \%)$ \\
\hline Acute coronary syndrome & $10(9.9 \%)$ & $10(9.9 \%)$ \\
\hline Palpitations/atrial fibrillation & $4(4.0 \%)$ & $4(4.0 \%)$ \\
\hline Uncontrolled hypo/hypertension & $1(1.0 \%)$ & $3(3.0 \%)$ \\
\hline Pulmonary oedema & $0(0.0 \%)$ & $1(1.0 \%)$ \\
\hline Knee surgery (all) & $5(5.0 \%)$ & $6(5.9 \%)$ \\
\hline Study knee & $2(2.0 \%)$ & $4(4.0 \%)$ \\
\hline Non-study knee & $3(3.0 \%)$ & $2(2.0 \%)$ \\
\hline
\end{tabular}

sunola oil with or without low-dose fish may confer a beneficial effect, but this unanticipated finding requires confirmation in further trials.

An alternative explanation is that both groups experienced a 'placebo effect'. Although the changes in pain scores in this study are comparable to those seen with 'placebo effect' for pain, ${ }^{40}$ this is difficult to assess in the current study due to lack of control group. The GAIT study, which compared glucosamine, chondroitin, glucosamine/chondroitin, celecoxib and placebo, demonstrated similar improvement in pain in all groups over 2 years. WOMAC pain scores declined in the first 12 weeks with little or no change thereafter. ${ }^{41}$ In our study, the WOMAC pain scores in both groups were similar at 3 months then began to diverge with participants in the low-dose group continuing to have reduction after 12 months. The initial reduction in WOMAC scores at 3 months is consistent with regression to the mean. However, it is unlikely that this phenomenon can fully explain the better outcome observed in the low-dose group during the second year of the study.

A study limitation is the lack of a control group. Inclusion of a small amount of fish oil allowed appropriate masking of the oils. This was successful as participants were not able to accurately detect the oil to which they had been randomised. The greater intolerance and greater withdrawal in the high-dose group was unexpected given the run-in period with daily ingestion of liquid oil and the additives of citrus oils, which gave both oils a similar taste. It was considered unethical to prevent fish oil supplements for 2 years in these older, more overweight participants and the $450 \mathrm{mg}$ EPA+DHA daily intake in the low-dose group complies with recommendations aimed at reducing cardiac mortality, which are based on the antiarrhythmic effect of these fatty acids. ${ }^{42}$ Anti-inflammatory effects have not been seen at doses this low. ${ }^{43}$

The question arises whether sunola oil may have some efficacy in OA. With hindsight, we believe that the most appropriate control group would have been no oil at all, which would of course sacrifice the ability to perform a blinded study. However, given the study we performed, it is a reasonable conclusion that it is still unknown whether low-dose fish oil and/or sunola oil are beneficial for knee OA.

This was an investigator-initiated rigorously conducted study with excellent 2-year follow-up. The strengths of the study 
include adequate masking of fish oil, repeated symptom measures and MRI imaging. There was a low overall dropout rate for the study despite significant withdrawal due to oil intolerance.

We found no benefit of high-dose fish oil supplementation compared with low-dose fish oil supplementation in knee OA. The unanticipated finding of better pain and function in the low-dose fish oil/sunola group requires further investigation.

Correction notice This article has been corrected since it was published Online First. The first name of the sixth author has been corrected and the affiliations for the sixth and last authors have been corrected.

Acknowledgement We would like to acknowledge the participants for their time and commitment to this study.

Contributors CLH, LMM, LGC and GJ designed the study and obtained funding. $\mathrm{RB}, \mathrm{KH}$ and TF recruited participants. CLH, LMM, GJ, RB, KH and TF screened participants. DA read and interpreted MR images. SEL provided statistical analysis and advice. CLH and MJ wrote the draft manuscript. CLH, GJ, LMM, SMP, LGC and MJ participated in data interpretation. All authors critically reviewed and edited the manuscript and approved the final version.

Funding Supported by National Health and Medical Research Council of Australia (Project Grant 451900), and Arthritis Australia.

Competing interests None declared.

Patient consent Obtained.

Ethics approval The Queen Elizabeth Hospital Human Research Ethics Committee Royal North Shore Hospital Human Research Ethics Committee, Tasmanian Human Research Ethics Committee.

Provenance and peer review Not commissioned; externally peer reviewed.

Data sharing statement Data is available for sharing on request to authors.

\section{REFERENCES}

1 Guccione AA, Felson DT, Anderson JJ, et al. The effects of specific medical conditions on the functional limitations of elders in the Framingham Study. Am $J$ Public Health 1994:84:351-8.

2 Hochberg MC, Altman RD, April KT, et al. American College of Rheumatology 2012 recommendations for the use of nonpharmacologic and pharmacologic therapies in osteoarthritis of the hand, hip, and knee. Arthritis Care Res (Hoboken) 2012;64:465-74

3 James MJ, Cleland LG. Dietary n-3 fatty acids and therapy for rheumatoid arthritis. Semin Arthritis Rheum 1997;27:85-97.

4 Proudman SM, James MJ, Spargo LD, et al. Fish oil in recent onset rheumatoid arthritis: a randomised, double-blind controlled trial within algorithm-based drug use. Ann Rheum Dis 2015;74:89-95.

5 Benito MJ, Veale DJ, FitzGerald 0 , et al. Synovial tissue inflammation in early and late osteoarthritis. Ann Rheum Dis 2005;64:1263-7.

6 Hill CL, Gale DG, Chaisson CE, et al. Knee effusions, popliteal cysts, and synovial thickening: association with knee pain in osteoarthritis. J Rheumatol 2001;28:1330-7.

7 Hill CL, Hunter DJ, Niu J, et al. Synovitis detected on magnetic resonance imaging and its relation to pain and cartilage loss in knee osteoarthritis. Ann Rheum Dis 2007;66:1599-603

8 Roemer FW, Guermazi A, Felson DT, et al. Presence of MRI-detected joint effusion and synovitis increases the risk of cartilage loss in knees without osteoarthritis at 30-month follow-up: the MOST study. Ann Rheum Dis 2011;70:1804-9.

9 Serhan CN, Chiang N, Van Dyke TE. Resolving inflammation: dual anti-inflammatory and pro-resolution lipid mediators. Nat Rev Immunol 2008:8:349-61.

10 Cleland LG, James MJ, Proudman SM. Fish oil: what the prescriber needs to know. Arthritis Res Ther 2006;8:202.

11 Curtis $\mathrm{CL}$, Hughes $\mathrm{CE}$, Flannery $\mathrm{CR}$, et al. n-3 fatty acids specifically modulate catabolic factors involved in articular cartilage degradation. I Biol Chem 2000:275:721-4.

12 Curtis $\mathrm{CL}$, Rees $\mathrm{SG}$, Little $\mathrm{CB}$, et al. Pathologic indicators of degradation and inflammation in human osteoarthritic cartilage are abrogated by exposure to $\mathrm{n}-3$ fatty acids. Arthritis Rheum 2002;46:1544-53.

13 Knott L, Avery NC, Hollander AP, et al. Regulation of osteoarthritis by omega-3 $(n-3)$ polyunsaturated fatty acids in a naturally occurring model of disease. Osteoarthritis Cartilage 2011;19:1150-7.

14 Wang Y, Wluka AE, Hodge AM, et al. Effect of fatty acids on bone marrow lesions and knee cartilage in healthy, middle-aged subjects without clinical knee osteoarthritis. Osteoarthritis Cartilage 2008;16:579-83.
15 Baker KR, Matthan NR, Lichtenstein AH, et al. Association of plasma n-6 and n-3 polyunsaturated fatty acids with synovitis in the knee: the MOST study. Osteoarthritis Cartilage 2012;20:382-7.

16 Adams J, Sibbritt D, Lui CW, et al. \{0mega\}-3 fatty acid supplement use in the 45 and Up Study Cohort. BMJ Open 2013;3.

17 Hill C, Gill TK, Appleton S, et al. The use of fish oil in the community: results of a population-based study. Rheumatology (Oxford) 2009;48:441-2.

18 National Heart Foundation of Australia (NHF). Position Statement: Fish, fish oils, n-3 polyunsaturated fatty acids and cardiovascular health (updated 2008). 2008.

19 Altman R, Asch E, Bloch D, et al. Development of criteria for the classification and reporting of osteoarthritis. Classification of osteoarthritis of the knee. Diagnostic and Therapeutic Criteria Committee of the American Rheumatism Association. Arthritis Rheum 1986:29:1039-49.

20 Altman RD, Gold GE. Atlas of individual radiographic features in osteoarthritis, revised. Osteoarthritis Cartilage 2007;15(Suppl 1):A1-A56.

21 Bellamy N. WOMAC Osteoarthritis Index User Guide. Version VIII. Brisbane, Australia, 2007.

22 Hawthorne G, Richardson J, Osborne R. The Assessment of Quality of Life (AQoL) instrument: a psychometric measure of health-related quality of life. Qual Life Res 1999:8:209-24.

23 Constant F, Guillemin F, Herbeth B, et al. Measurement methods of drug consumption as a secondary judgment criterion for clinical trials in chronic rheumatic diseases. Am J Epidemiol 1997;145:826-33.

24 Jones $\mathrm{G}$, Glisson $\mathrm{M}$, Hynes $\mathrm{K}$, et al. Sex and site differences in cartilage development: a possible explanation for variations in knee osteoarthritis in later life. Arthritis Rheum 2000;43:2543-9.

25 Dore D, Quinn S, Ding C, et al. Natural history and clinical significance of MRI-detected bone marrow lesions at the knee: a prospective study in community dwelling older adults. Arthritis Res Ther 2010;12:R223.

26 Laslett LL, Dore DA, Quinn SJ, et al. Zoledronic acid reduces knee pain and bone marrow lesions over 1 year: a randomised controlled trial. Ann Rheum Dis 2012;71:1322-8

27 James MJ, Ursin VM, Cleland LG. Metabolism of stearidonic acid in human subjects: comparison with the metabolism of other $n-3$ fatty acids. Am I Clin Nutr 2003;77:1140-5.

28 Liu GF, Lu K, Mogg R, et al. Should baseline be a covariate or dependent variable in analyses of change from baseline in clinical trials? Stat Med 2009;28:2509-30.

29 R Core Team. R: A language and environment for statistical computing. 2013. http://www.r-project.org/ (cited 3 Sep 3 2014)

30 Pinheiro J, Bates D, DebRoy S, et al. R Core Team. nlme: Linear and Nonlinear Mixed Effects Models. 2014. http://cran.r-project.org/web/packages/nlme/index.html (cited 3 Sep 3 2014).

31 Honaker J, King G, Blackwell M. Amelia II: a program for missing data. J Stat Software 2011:45:1-47.

32 Tingley D, Yamamoto T, Hirose K, et al. Mediation: R Package for Causal Mediation Analysis. J Stat Software 2014:59:1-38.

33 Nguyen TV, Eisman JA. Assessment of significant change in BMD: a new approach. J Bone Miner Res 2000;15:369-72.

34 Christensen $\mathrm{R}$, Henriksen M, Leeds AR, et al. The effect of weight maintenance on symptoms of knee osteoarthritis in obese patients: 12 month randomized controlled trial. Arthritis Care Res (Hoboken) 2015;67:640-50.

35 Messier SP, Mihalko SL, Legault C, et al. Effects of intensive diet and exercise on knee joint loads, inflammation, and clinical outcomes among overweight and obese adults with knee osteoarthritis: the IDEA randomized clinical trial. JAMA 2013:310:1263-73.

36 Riddle DL, Stratford PW. Body weight changes and corresponding changes in pain and function in persons with symptomatic knee osteoarthritis: a cohort study. Arthritis Care Res (Hoboken) 2013;65:15-22.

37 Stammers T, Sibbald B, Freeling P. Efficacy of cod liver oil as an adjunct to non-steroidal anti-inflammatory drug treatment in the management of osteoarthritis in general practice. Ann Rheum Dis 1992;51:128-9.

38 Grompone MA. Sunflower oil. In: Shahidi F, ed. Bailey's Industrial Oil and Fat Products. 6th edn. John Wiley \& Sons, Inc, 2005:655-730.

39 Visioli F, Bellomo G, Galli C. Free radical-scavenging properties of olive oil polyphenols. Biochem Biophys Res Commun 1998;247:60-4.

40 Zhang W, Robertson J, Jones AC, et al. The placebo effect and its determinants in osteoarthritis: meta-analysis of randomised controlled trials. Ann Rheum Dis 2008:67:1716-23.

41 Sawitzke AD, Shi H, Finco MF, et al. Clinical efficacy and safety of glucosamine, chondroitin sulphate, their combination, celecoxib or placebo taken to treat osteoarthritis of the knee: 2-year results from GAIT. Ann Rheum Dis 2010;69:1459-64.

42 Flock MR, Harris WS, Kris-Etherton PM. Long-chain omega-3 fatty acids: time to establish a dietary reference intake. Nutr Rev 2013;71:692-707.

43 Calder PC. Omega-3 polyunsaturated fatty acids and inflammatory processes: nutrition or pharmacology? Br J Clin Pharmacol 2013;75:645-62. 